\title{
Empiric guideline-recommended weight-based vancomycin dosing and mortality in methicillin- resistant Staphylococcus aureus bacteremia: $a$ retrospective cohort study
}

\author{
Ronald G Hall II,2*, Christopher A Giuliano ${ }^{3,9}$, Krystal K Haase ${ }^{3}$, Kathleen A Hazlewood ${ }^{1,7}$, Chistopher R Frei ${ }^{4}$, \\ Nicolas A Forcade ${ }^{4,10}$, Sara D Brouse ${ }^{1,8}$, Todd Bell ${ }^{5}$, Roger J Bedimo ${ }^{6}$ and Carlos A Alvarez ${ }^{1,2}$
}

\begin{abstract}
Background: No studies have evaluated the effect of guideline-recommended weight-based dosing on in-hospital mortality of patients with methicillin-resistant Staphylococcus aureus bacteremia.

Methods: This was a multicenter, retrospective, cohort study of patients with methicillin-resistant Staphylococcus aureus bacteremia receiving at least 48 hours of empiric vancomycin therapy between 01/07/2002 and 30/06/2008. We compared in-hospital mortality for patients treated empirically with weight-based, guideline-recommended vancomycin doses (at least $15 \mathrm{mg} / \mathrm{kg} /$ dose) to those treated with less than $15 \mathrm{mg} / \mathrm{kg} / \mathrm{dose}$. We used a general linear mixed multivariable model analysis with variables identified a priori through a conceptual framework based on the literature.

Results: A total of 337 patients who were admitted to the three hospitals were included in the cohort. One-third of patients received vancomycin empirically at the guideline-recommended dose. Guideline-recommended dosing was not associated with in-hospital mortality in the univariable (16\% vs. 13\%, OR 1.26 [95\%Cl 0.67-2.39]) or multivariable (OR $0.71,95 \% \mathrm{Cl} 0.33-1.55)$ analysis. Independent predictors of in-hospital mortality were ICU admission, Pitt bacteremia score of 4 or greater, age 53 years or greater, and nephrotoxicity.
\end{abstract}

Conclusions: Empiric use of weight-based, guideline-recommended empiric vancomycin dosing was not associated with reduced mortality in this multicenter study.

Keywords: Weight, Obesity, Efficacy, MRSA, Vancomycin, Bacteremia

\section{Background}

Vancomycin is commonly used for the treatment of methicillin-resistant Staphylococcus aureus (MRSA) bacteremia. The United States of America Food and Drug Administration (FDA) originally approved a dosing regimen of 1 gram administered intravenously (IV) every 12 hours (or $500 \mathrm{mg}$ IV every six hours). The FDA approved dosing regimen has not changed in over 50 years despite several studies demonstrating that vancomycin pharmacokinetics

\footnotetext{
* Correspondence: ronald.hall@ttuhsc.edu

'Department of Pharmacy Practice, Texas Tech University Health Sciences

Center, School of Pharmacy, Dallas, USA

2Department of Clinical Sciences, University of Texas Southwestern, Dallas, USA

Full list of author information is available at the end of the article
}

in adults are best predicted by actual body weight [1-4]. Dosing handbooks that are used in clinical practice continue to recommend a fixed dose for all patients regardless of weight $[5,6]$.

Over the past decade, there has been an increase in the incidence of MRSA strains with vancomycin minimum inhibitory concentration (MIC) values $\geq 1 \mu \mathrm{g} / \mathrm{ml}$ as well as heteroresistant vancomycin-intermediate $S$. aureus. In response to increasing vancomycin MICs in MRSA isolates, the Infectious Diseases Society of America, the American of Society of Health-Systems Pharmacists, and the Society of Infectious Diseases Pharmacists developed weight-based dosing recommendations for vancomycin based on pharmacokinetic and pharmacodynamic data $(15-20 \mathrm{mg} / \mathrm{kg} /$

\section{Biomed Central}


dose IV administered every eight to 12 hours) [7]. The clinical effectiveness of this empiric weight-based, guideline-recommended dosing regimen (at least $15 \mathrm{mg} / \mathrm{kg} /$ dose) has yet to be critically evaluated.

Data evaluating guideline-recommended weight-based vancomycin dosing are needed to confirm the efficacy of this approach versus lower traditional dosing. Therefore, we conducted a multicenter retrospective cohort study to evaluate the effectiveness of guideline-recommended weight-based dosing for vancomycin on mortality of patients with MRSA bacteremia.

\section{Methods}

\section{Study location and patients}

We identified a retrospective cohort of patients admitted with MRSA bacteremia (using microbiological records) between $01 / 07 / 2002$ and 30/06/2008 at three types of hospitals (400 bed urban, 200 bed veteran affairs, and 604 bed university). Patients were included if they were at least 18 years old and had received parenteral vancomycin for at least 48 hours. Patients were excluded if, at the time of the first vancomycin dose, they were receiving dialysis, had a creatinine clearance of $30 \mathrm{ml} / \mathrm{min}$ or less based upon the Cockcroft-Gault equation, received prior vancomycin within the hospital stay, had a culture-proven MRSA infection in the previous six months, or were pregnant [8]. The institutional review board of each respective site (North Texas Veterans Health Care System, Texas Tech University Health Sciences Center, and University of Texas Health Science Center, San Antonio) approved the study and waived the need for informed consent.

\section{Study design and data collection}

We conducted a retrospective cohort study of patients who received guideline-recommended weight-based dosing with those receiving lower dosing of vancomycin for MRSA bacteremia. Our primary outcome was in-hospital mortality.

\section{Study definitions}

All definitions were selected prospectively during the initial trial design. Guideline-recommended weightbased dosing was defined as at least $30 \mathrm{mg} / \mathrm{kg} /$ day in the first 24 hours (at least $15 \mathrm{mg} / \mathrm{kg} /$ day for patients with a creatinine clearance of $30-50 \mathrm{ml} / \mathrm{min}$ ). Lower dosing was defined as receiving less than $30 \mathrm{mg} / \mathrm{kg} /$ day (less than $15 \mathrm{mg} / \mathrm{kg} /$ day for patients with a creatinine clearance of $30-50 \mathrm{ml} / \mathrm{min}$ ). Pitt bacteremia score was calculated based on the date when the first positive blood culture was obtained $[9,10]$. In-hospital mortality was defined as patient death occurring within the index hospital stay. Nephrotoxicity was defined as an increase in creatinine by more than $0.5 \mathrm{mg} / \mathrm{dl}$ or greater than a $50 \%$ increase from baseline on two consecutive days [7].

\section{Statistical analysis}

Recursive partitioning was used to ascertain significant cut-points in continuous candidate variables associated with an increased risk of mortality [11]. Univariable associations were explored using either Chi-square or Fisher's Exact tests. A Pitt bacteremia score cutoff of 4 or higher was used based on previous literature demonstrating significantly higher sensitivity and specificity for predicting severity of illness [12]. A vancomycin trough $15 \mathrm{mcg} / \mathrm{ml}$ or greater was based on the guideline recommended trough concentration range of $15-20 \mathrm{mcg} / \mathrm{ml} \mathrm{[7].} \mathrm{Variables} \mathrm{exam-}$ ined in the initial univariable analysis included receipt of guideline recommended weight-based vancomycin dosing, intensive care unit (ICU) admission, age 53 years, Pitt bacteremia score of 4 or higer, vancomycin trough $15 \mathrm{mcg} / \mathrm{ml}$ or greater, nephrotoxicity, Charlson comorbidity index score of 5 or higher, weight of $100 \mathrm{~kg}$ or greater, and gender.

Variables identified as significant through univariable analysis $(p<0.1)$ and those conceptually regarded as biologically reasonable causes of mortality were considered for inclusion in the multivariable model. A generalized linear mixed-effect model was utilized to identify independent predictors of mortality. Hospital site was treated as a random effect whereas other covariates were treated as fixed effects. Variables were retained in the multivariable model if their respective $\mathrm{p}$ values were $<0.05$. Adjusted ORs and $95 \%$ confidence intervals were calculated for each variable. Effect measure modification and biologic interaction were also extensively evaluated. All analyses were performed using SAS 9.2 (Cary, North Carolina) and RTREE (Available at: http://c2s2.yale.edu/software. rtree).

\section{Results \\ Patients}

A total of 798 patients with MRSA bacteremia were evaluated, with 337 included in the study cohort after application of the exclusion criteria. In-hospital mortality data were not collected for one patient. The baseline characteristics of the cohort are shown in Table 1. Forty-seven patients $(14 \%)$ died during their hospital stay. Survivors were more likely to be male and less likely to have vancomycin started in the ICU (34 vs. 83\%). Survivors also had a younger median age, higher baseline renal function, lower Charlson Comorbidity Index, and a less severe Pitt Bacteremia score. Vancomycin was empirically dosed according to guidelines in 33\% of survivors and 38\% of non-survivors $(p=0.48)$ with a median initial daily dose of 24.9 vs. $21.0 \mathrm{mg} / \mathrm{kg} /$ day $(p=0.036)$, respectively. Vancomycin was adjusted for renal function in 39\% of survivors and $59 \%$ of non-survivors $(p=0.028)$. 
Table 1 Baseline characteristics of the cohort ${ }^{A}$

\begin{tabular}{|c|c|c|c|}
\hline Characteristic & Survivors $(n=289)$ & Non-survivors $(n=47)$ & $p$-value \\
\hline Male gender (\%) & $77 \%$ & $94 \%$ & 0.009 \\
\hline Age (years) & $53(42,63)$ & $65(56,77)$ & $<0.001$ \\
\hline Race (\%) & & & 0.66 \\
\hline Caucasian & $64 \%$ & $74 \%$ & \\
\hline African American & $15 \%$ & $11 \%$ & \\
\hline Hispanic & $18 \%$ & $13 \%$ & \\
\hline Other & $3 \%$ & $2 \%$ & \\
\hline Height $(\mathrm{cm})$ & $175.0(165.1,180.3)$ & $175.0(169.5,183.0)$ & 0.14 \\
\hline Weight (kg) & $78.1(65,95.6)$ & $80.0(63.0,99.0)$ & 0.91 \\
\hline Serum Creatinine (mg/dl) & $0.90(0.7,1.2)$ & $1.10(0.7,1.5)$ & 0.035 \\
\hline Creatinine Clearence (ml/min) & $86.9(61.0,122)$ & $64.0(42.9,86.3)$ & $<0.001$ \\
\hline Pitt Bacteremia Score & $1(0,3)$ & $3(2,6)$ & $<0.001$ \\
\hline Charlson Comorbidity Index & $2(1,3)$ & $2(2,4)$ & 0.030 \\
\hline Guideline-recommended Vancomycin Dose Received (\%) & $33 \%$ & $38 \%$ & 0.48 \\
\hline Dose adjusted for renal function (\%) & $39 \%$ & $59 \%$ & 0.028 \\
\hline Vancomycin Started in Intensive Care Unit (\%) & $34 \%$ & $83 \%$ & $<0.001$ \\
\hline Initial Vancomycin Dose (mg/kg/day) & $24.9(19.6,30.4)$ & $21.0(17.0,29.0)$ & 0.036 \\
\hline \multicolumn{4}{|l|}{ Nephrotoxins (\%) } \\
\hline Intravenous Contrast & $34 \%$ & $13 \%$ & 0.004 \\
\hline Aminoglycosides & $19 \%$ & $19 \%$ & 0.98 \\
\hline Vasopressors & $7 \%$ & $36 \%$ & $<0.001$ \\
\hline Infection source (\%) & & & $<0.001$ \\
\hline Bloodstream catheter-related & $18 \%$ & $36 \%$ & \\
\hline Central nervous system & $0.4 \%$ & $0 \%$ & \\
\hline Gastrointestinal & $0.7 \%$ & $0 \%$ & \\
\hline Osteomyelitis & $0.7 \%$ & $4 \%$ & \\
\hline Pulmonary & $15 \%$ & $36 \%$ & \\
\hline Skin/muscle & $36 \%$ & $6 \%$ & \\
\hline Other & $20 \%$ & $17 \%$ & \\
\hline
\end{tabular}

$\mathrm{A}=$ Results are presented as median (interquartile range) unless otherwise noted.

The primary sources of bacteremia were skin/soft tissue (32.5\%), intravenous catheter (20.2\%), and pulmonary (17.8\%). Other documented sources included genitourinary $(8 \%)$, bone $(1.2 \%)$, gastrointestinal $(0.5 \%)$, and central nervous system $(0.3 \%)$. The source of bacteremia was not specified for $19.8 \%$ of patients. Non-survivors were less likely to have a skin/muscle source of their bacteremia and more likely to have a bloodstream catheter-related or pulmonary source. Seventy-nine patients $(23.4 \%)$ received concomitant antimicrobials active against MRSA. Non-survivors were less likely to receive IV contrast dye and more likely to receive vasopressors. Nineteen percent of both groups received aminoglycosides. Nephrotoxicity occurred in 82 patients $(23.4 \%)$.

The overall median (interquartile range) length of hospital stay was $16(9,32)$ days for survivors and $23(14,56)$ for non-survivors $(p=0.009)$. The median length of stay after vancomycin initiation was $14(7.5,26.5)$ days for survivors and $19(9,42)$ for non-survivors. Thirty-two percent of patients had a hospital stay longer than 28 days. The median ICU length of stay was $0(0,4)$ days for survivors and $9(3,39)$ for non-survivors.

\section{Univariable and multivariable analysis}

In the univariable analysis (Table 2), mortality was similar among patients who received guideline-recommended dosing versus lower dosing (16\% vs. 13\%, OR 1.26 [95\%CI $0.67-2.39])$. Factors that increased risk for mortality by univariable analysis included: male gender, age of 53 years or greater, ICU admission, Pitt bacteremia score of four or greater, nephrotoxicity, and vancomycin trough of 15 $\mathrm{mcg} / \mathrm{ml}$ or greater.

Vancomycin dosing also was not significantly associated with mortality (OR 1.05, 95\%CI 0.48-2.27) when retained in the multivariable analysis (Table 3 ). The only factors that remained significant in the multivariable model were 
Table 2 Univariable analysis of risk factors for mortality

\begin{tabular}{lcc}
\hline Variable & Odds Ratio & $\begin{array}{c}\text { 95\% Confidence } \\
\text { Interval }\end{array}$ \\
\hline $\begin{array}{l}\text { Guideline-Recommended } \\
\text { Vancomycin Dosing }\end{array}$ & 1.26 & $0.67-2.39$ \\
\hline Intensive Care Unit Admission & 9.50 & $4.27-21.12$ \\
\hline Age of 53 years or greater & 7.89 & $3.04-20.52$ \\
\hline $\begin{array}{l}\text { Pitt Bacteremia Score of four } \\
\text { or greater }\end{array}$ & 4.47 & $2.34-8.54$ \\
\hline $\begin{array}{l}\text { Vancomycin trough of } \\
15 \text { mcg/ml or greater }\end{array}$ & 2.48 & $1.30-4.74$ \\
\hline $\begin{array}{l}\text { Nephrotoxicity } \\
\text { Charlson Comorbidity Index } \\
\text { of five or greater }\end{array}$ & 4.95 & $2.60-9.42$ \\
\hline $\begin{array}{l}\text { Weight of } 100 \text { kg or greater } \\
\text { Male gender }\end{array}$ & 1.63 & $0.75-3.54$ \\
\hline
\end{tabular}

age of 53 years or greater, ICU admission, Pitt bacteremia score of four or greater, and nephrotoxicity.

\section{Discussion}

To our knowledge, this is the first published multicenter study to evaluate the effect of empiric guidelinerecommended weight-based dosing on mortality in MRSA bacteremia. We did not observe any significant relationship between empiric guideline-recommended weight-based vancomycin dosing and in-hospital mortality. Independent predictors of in-hospital mortality were ICU admission, Pitt bacteremia score of 4 or greater, age 53 years or greater, and nephrotoxicity.

Most studies evaluating the effectiveness of vancomycin dosing for MRSA infections have compared high vs. low vancomycin trough concentrations. Our results parallel the findings of these studies. A recently published prospective cohort study assessing patients with MRSA pneumonia and bacteremia failed to demonstrate that achieving vancomycin trough concentrations to 4 to 5 times the MIC were associated with any difference in clinical response rates [13]. Another study of 102 patients

Table 3 Multivariable analysis of independent risk factors for mortality

\begin{tabular}{lcc}
\hline Variable & Odds Ratio & $\begin{array}{c}\mathbf{9 5 \%} \text { Confidence } \\
\text { interval }\end{array}$ \\
\hline $\begin{array}{l}\text { Guideline-Recommended } \\
\text { Vancomycin Dosing }\end{array}$ & 0.71 & $0.33-1.55$ \\
\hline Intensive Care Unit Admission & 6.14 & $2.46-15.35$ \\
\hline Age of 53 years or greater & 5.58 & $1.88-16.51$ \\
\hline $\begin{array}{l}\text { Pitt Bacteremia Score of four } \\
\text { or greater }\end{array}$ & 2.93 & $1.21-7.09$ \\
\hline Nephrotoxicity & 2.29 & $1.05-4.97$ \\
\hline $\begin{array}{l}\text { Vancomycin trough of } \\
15 \text { mcg/ml or greater }\end{array}$ & 1.24 & $0.56-2.75$ \\
\hline
\end{tabular}

evaluated the association between vancomycin trough concentrations and mortality in MRSA pneumonia; 11\% of patients had concomitant bacteremia [14]. This study did not find a difference in vancomycin trough concentrations between survivors and non-survivors (13.6 vs. $13.9 \mathrm{mcg} / \mathrm{ml}$ ). Using vancomycin trough concentrations to evaluate mortality is potentially confounded by the fact that vancomycin is eliminated by the kidneys. Acute renal failure is an independent risk factor for mortality, which also results in increased vancomycin trough concentrations (regardless of the cause of acute renal failure).

Our findings suggest that other patient factors play are more important factors in determining in-hospital mortality for patients with MRSA bacteremia than vancomycin dosing. The empiric use of guideline-recommended weight-based dosing in the absence of compelling efficacy or effectiveness data has the potential to place patients at an increased risk of concentration-related adverse events with an uncertain benefit to the patient. Clinicians should critically evaluate vancomycin trough concentrations and kidney function to help minimize this risk in patients receiving guideline-recommended weight-based vancomycin regimens.

The implications of this study on clinical practice are limited by its retrospective design, use of in-hospital mortality, lack of MIC data, and lack of data regarding time to a therapeutic vancomycin trough concentration. Utilizing in-hospital mortality as our primary endpoint resulted in fewer events than utilizing 30 day mortality or vancomycin failure [15-17]. This difference created greater variability in the multivariable model and limited the number of candidate variables evaluated in the multivariable model. In spite of this limitations, the factors associated with mortality in this study are similar to others [15]. The study's retrospective design allowed a more realistic estimate of clinical effectiveness than could be observed in a randomized, controlled trial. Increased vancomycin MIC values may have also played a role in the lack of benefit associated with guidelinerecommended dosing. However, clinicians rarely have vancomycin MIC data available when selecting an empiric vancomycin dose. We did not collect data regarding the time to the first therapeutic vancomycin trough, as quicker times to therapeutic trough concentrations may improve vancomycin efficacy. However, this information may have been biased in this retrospective evaluation by clinician judgment regarding when trough concentrations were ordered. Our results may have also been subject to a selection bias that patients weighing greater than 70 kilograms were less likely to receive guideline-recommended, weight-based vancomycin dosing since most patients (85\%) received 1 gram every 12 hours. Furthermore, the implications of loading doses on 
mortality need further evaluation since none of the study institutions utilized this dosing strategy.

\section{Conclusions}

In our study, we did not observe any significant association between guideline-recommended dosing and mortality. These results, in concert with the findings of others, call for a prospective, randomized comparison of empiric use of guideline-recommended weight-based vs. traditional dosing of vancomycin to ensure that patients treated with vancomycin receive doses that maximize efficacy and safety.

\section{Competing interests}

Grant funding from AstraZeneca, Ortho-McNeil Janssen, and Pfizer: CRF, Scientific Advisory Board for Tibotec Therapeutics and Gilead Sciences: RJB, None: $\mathrm{RGH}, \mathrm{CAA}, \mathrm{CAG}, \mathrm{KKH}, \mathrm{KAH}, \mathrm{NAF}, \mathrm{SDB}$, TB

\section{Authors' contributions}

$\mathrm{RGH}, \mathrm{CAG}, \mathrm{KKH}, \mathrm{KAH}, \mathrm{SDB}, \mathrm{RJB}$ were involved in the study concept and design. $\mathrm{RGH}, \mathrm{CAG}, \mathrm{CAA}, \mathrm{CRF}$ were involved in the data analysis and interpretation. $\mathrm{RGH}, \mathrm{CAG}, \mathrm{KKH}, \mathrm{KAH}, \mathrm{CAA}, \mathrm{CRF}, \mathrm{NAF}, \mathrm{SDB}, \mathrm{TB}, \mathrm{RJB}$ were involved in the drafting of the manuscript for important intellectual content and had final approval of the manuscript

\section{Acknowledgements}

Dr. Hall and Alvarez's effort was supported by Grant Number KL2RR024983, titled, "North and Central Texas Clinical and Translational Science Initiative" (Milton Packer, M.D., PI) from the National Center for Research Resources (NCRR), a component of the National Institutes of Health $(\mathrm{NIH})$, and $\mathrm{NIH}$ Roadmap for Medical Research, and its contents are solely the responsibility of the authors and do not necessarily represent the official views of the NCRR or NIH. Information on NCRR is available at http://www.ncrr.nih.gov/. Information on Re-engineering the Clinical Research Enterprise can be obtained from http://nihroadmap.nih.gov/clinicalresearch/overviewtranslational.asp. Dr. Frei's time was supported by NIH grant KL2RR025766. The NIH had no role in the study design, data collection, analysis/ interpretation, manuscript drafting, or submission.

\section{Author details}

'Department of Pharmacy Practice, Texas Tech University Health Sciences Center, School of Pharmacy, Dallas, USA. ${ }^{2}$ Department of Clinical Sciences, University of Texas Southwestern, Dallas, USA. ${ }^{3}$ Department of Pharmacy Practice, Texas Tech University Health Sciences Center, School of Pharmacy, Amarillo, USA. ${ }^{4}$ Division of Pharmacotherapy, University of Texas, Austin, USA. ${ }^{5}$ Department of Internal Medicine, Texas Tech University Health Sciences Center, School of Medicine, Amarillo, USA. ${ }^{6}$ University of Texas Southwestern, Department of Internal Medicine, Dallas, USA. ${ }^{7}$ University of Wyoming School of Pharmacy, Swedish Family Medicine Residency Program, Englewood, USA. ${ }^{8}$ University of Kentucky Healthcare, Lexington, USA. ${ }^{9}$ Wayne State University Eugene Applebaum College of Pharmacy and Health Sciences, Detroit, USA.

${ }^{10}$ Mission Regional Medical Center, Mission, USA.

Received: 2 December 2011 Accepted: 27 April 2012

Published: 27 April 2012

\section{References}

1. Ducharme MP, Slaughter RL, Edwards DJ: Vancomycin pharmacokinetics in a patient population: effect of age, gender, and body weight. Ther Drug Monit 1994, 16:513-518.

2. Blouin RA, Bauer LA, Miller DD, Record KE, Griffen WO Jr: Vancomycin pharmacokinetics in normal and morbidly obese subjects. Antimicrob Agents Chemother 1982, 21:575-580.

3. Bauer LA, Black DJ, Lill JS: Vancomycin dosing in morbidly obese patients. Eur J Clin Pharmacol 1998, 54:621-625

4. Vance-Bryan K, Guay DR, Gilliland SS, Rodvold KA, Rotschafer JC: Effect of obesity on vancomycin pharmacokinetic parameters as determined by using a Bayesian forecasting technique. Antimicrob Agents Chemother 1993, 37:436-440.

5. Bartlett B: Pocket Book of Infectious Disease Therapy. 12th edition. Philidelphia: Lippincott Williams \& Wilkins; 2004:2004

6. Gilbert DN, Moellering RC, Eliopoulos GM, Chambers HF, Saag MS: The Sanford guide to antimicrobial therapy. Sperryville, VA: Antimicrobial Therapy, Inc; 2011

7. Rybak M, Lomaestro B, Rotschafer JC, Moellering R Jr, Craig W, Billeter M, Dalovisio JR, Levine DP: Therapeutic monitoring of vancomycin in adult patients: a consensus review of the American Society of Health-System Pharmacists, the Infectious Diseases Society of America, and the Society of Infectious Diseases Pharmacists. Am J Health Syst Pharm 2009, 66:82-98.

8. Cockcroft DW, Gault MH: Prediction of creatinine clearance from serum creatinine. Nephron 1976, 16:31-41

9. Chow JW, Fine MJ, Shlaes DM, Quinn JP, Hooper DC, Johnson MP, Ramphal R, Wagener MM, Miyashiro DK, Yu VL: Enterobacter bacteremia: clinical features and emergence of antibiotic resistance during therapy. Ann Intern Med 1991, 115:585-590.

10. Chow JW, Yu VL: Combination antibiotic therapy versus monotherapy for gram-negative bacteraemia: a commentary. Int J Antimicrob Agents 1999, 11:7-12.

11. Zhang $\mathrm{H}$, Singer B: Recursive partitioning in the health sciences. New York: Springer; 1999.

12. Rhee JY, Kwon KT, Ki HK, Shin SY, Jung DS, Chung DR, Ha BC, Peck KR, Song $\mathrm{H}$ : Scoring systems for prediction of mortality in patients with intensive care unit-acquired sepsis: a comparison of the Pitt bacteremia score and the Acute Physiology and Chronic Health Evaluation II scoring systems. Shock 2009, 31:146-150.

13. Hidayat LK, Hsu DI, Quist R, Shriner KA, Wong-Beringer A: High-dose vancomycin therapy for methicillin-resistant Staphylococcus aureus infections: efficacy and toxicity. Arch Intern Med 2006, 166:2138-2144.

14. Jeffres MN, Isakow W, Doherty JA, McKinnon PS, Ritchie DJ, Micek ST, Kollef $\mathrm{MH}$ : Predictors of Mortality for Methicillin-Resistant Staphylococcus aureus Health-Care-Associated Pneumonia: Specific Evaluation of Vancomycin Pharmacokinetic Indices. Chest 2006, 130:947-955.

15. Wang FD, Chen YY, Chen TL, Liu CY: Risk factors and mortality in patients with nosocomial Staphylococcus aureus bacteremia. Am J Infect Control 2008, 36:118-122.

16. Lodise TP, Graves J, Evans A, Graffunder E, Helmecke M, Lomaestro BM, Stellrecht K: Relationship between vancomycin MIC and failure among patients with methicillin-resistant Staphylococcus aureus bacteremia treated with vancomycin. Antimicrob Agents Chemother 2008, 52:3315-3320.

17. Kullar R, Davis SL, Levine DP, Rybak MJ: Impact of vancomycin exposure on outcomes in patients with methicillin-resistant Staphylococcus aureus bacteremia: support for consensus guidelines suggested targets. Clin Infect Dis 2011, 52:975-981.

doi:10.1186/1471-2334-12-104

Cite this article as: Hall et al:: Empiric guideline-recommended weightbased vancomycin dosing and mortality in methicillin-resistant Staphylococcus aureus bacteremia: a retrospective cohort study. BMC Infectious Diseases 2012 12:104.

\section{Submit your next manuscript to BioMed Central and take full advantage of:}

- Convenient online submission

- Thorough peer review

- No space constraints or color figure charges

- Immediate publication on acceptance

- Inclusion in PubMed, CAS, Scopus and Google Scholar

- Research which is freely available for redistribution 